Journal of Computer Science 3 (4): 233-237, 2007

ISSN 1549-3636

(C) 2007 Science Publications

\title{
Seamless Handoff Using Mailbox Approach
}

\author{
Manpreet Singh and Rajneesh Gujral \\ Department of Computer Engineering, M. M. Engineering College, Mullana-133203, Haryana, India
}

\begin{abstract}
Mobile IP is used to keep track of location information and make the data available to the mobile devices anytime, anywhere. Mobile IP has been widely accepted but lacks in providing seamless handoff. We have proposed a framework for wireless network that uses a flexible and adaptive mailbox-based scheme. In this scheme a mailbox associated with each mobile node while allowing de coupling between them. The FIFO message buffer of mailbox used to store incoming messages destined to a mobile node. Mailbox can be detached from its owner node in the sense that the mailbox can reside at a location different from the current location of the owner node. During handoff, mailbox of mobile node can itself decide whether it has to move from current mobility agent (home agent or foreign agent) to new mobility agent. A pull technique, MPUL (Message Pull) adopted to implement the message delivery from mailbox to its owner node. The performance tradeoff for various mobility conditions are evaluated using analytical model.
\end{abstract}

Key words: Handoff, mobility agent (home agent or foreign agent), mailbox, care of address (CoA), pull technique, owner node

\section{INTRODUCTION}

Mobile IP is used to keep track of location information and make the data available to the mobile devices anytime, anywhere ${ }^{[1]}$. The goal of Mobile IP is to provide a host the ability to stay connected to the Internet regardless of its location ${ }^{[2,3]}$. Mobile IP network components include Mobile Node (MN), home agent, foreign agent, access routers, Correspondent Node (CN).In Mobile IP, the movement of a MN from one network to another introduces mobility agents such as the home agent (HA) and foreign agent (FA).Mobility Agents (MAs) advertise their presence via agent advertisement messages so that they become known by the MN. A MN may optionally solicit agent advertisement messages from any locally attached MAs through an agent solicitation message and receive agent advertisements. Then it determines whether it is on its home network or on a foreign network. When a MN detects that it has moved to a foreign network, it obtains a care-of address (CoA) on the foreign network. The CoA can be determined either from FA advertisements or by some external assignment mechanism such as Dynamic Host Configuration Protocol (DHCP). The message from correspondent node to $\mathrm{MN}$ is first routed with regular IP routing to its home network where they are captured by the home agent. The home agent then tunnels these

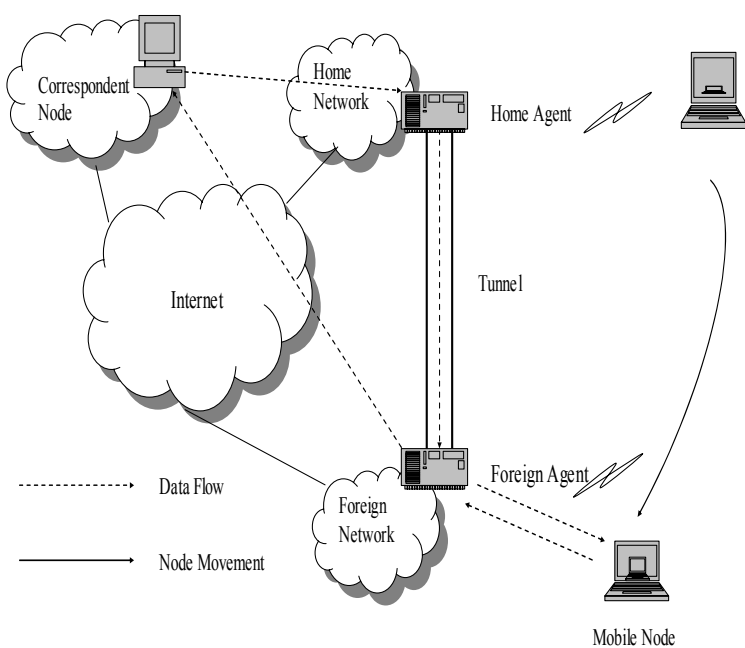

Fig. 1: Mobile IP operation

packets to the foreign agent using CoA which, in turn, forwards them to the final destination as shown in Fig. 1. An extension to the registration process, called seamless handoff ${ }^{[4,5]}$, enables foreign agents to also make use of binding updates to reduce packet loss during a handoff. However, Mobile IP suffers from the well known triangle routing and $2 \mathrm{x}$ problem. Therefore, Mobile IP route optimization ${ }^{[6,7]}$ has been proposed to alleviate this problem.

Corresponding Author: $\quad$ Manpreet Singh, Department of Computer Engineering, M. M. Engineering College, Mullana133203, Haryana, India 
Another problem with Mobile IP is that each time a MN changes its location it has to register its $\mathrm{CoA}$ at home agent. This causes much signaling traffic between $\mathrm{MN}$ and its home agent especially when the registration lifetime is short and $\mathrm{MN}$ is moving fast. During this handoff period some packets are lost and some are out of order ${ }^{[8,9]}$.

We have proposed a framework that uses a flexible and adaptive mailbox-based scheme, in which a mailbox is associated with each mobile node while allowing the de coupling between them. The FIFO message buffer of mailbox is used to store incoming messages destined to a mobile node can be detached from its owner node in the sense that the mailbox can reside at a location different from the current location of the owner node. During handoff, mailbox of mobile node can itself decide whether it has to move itself from current mobility agent (home agent or foreign agent) to new mobility agent. De coupling of mailbox from its owner results in adaptive location management that enables dynamic tradeoff between the packet delivery cost and the registration cost thereby minimizing the total cost. In pull technique the MN keeps the address of its mailbox and retrieves message from the mailbox whenever needed. The mailbox does not need to know the MN current location and therefore location registration is avoided.

Generic framework design: The generic framework uses a flexible and adaptive mailbox-based scheme, which associates a mailbox with each mobile node while allowing de coupling between them. Communication between $\mathrm{CN}$ and $\mathrm{MN}$ is usually two fold as shown in Fig. 2.

Step1: Sender routes the packet to receiver's mailbox.

Step2: Receiver receives the packet from its mailbox using pull technique.

However if both sender and receiver are on same network then messages will be directly routed to the receiver. Initially the mailbox resides on the same mobility agent as its owner. On crossing the network boundary and after receiving the $\mathrm{CoA}$ from new foreign agent it sends a registration message to old foreign agent (oFA) where the mailbox is currently residing.

The old foreign agent in lieu of registration message activates the mailbox operation by passing a control message to it.

Architecture of mailbox: By incorporating a decision capability regarding its migration to new foreign agent (nFA), the mailbox is implemented as an intelligent system and the factors affecting this decision are the

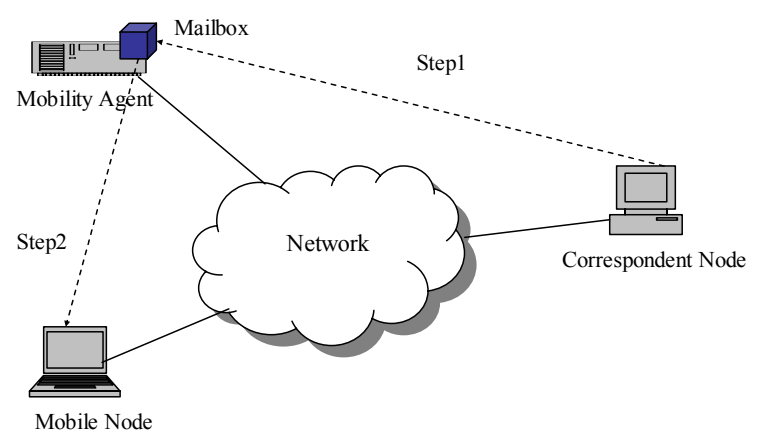

Fig. 2: Mailbox approach

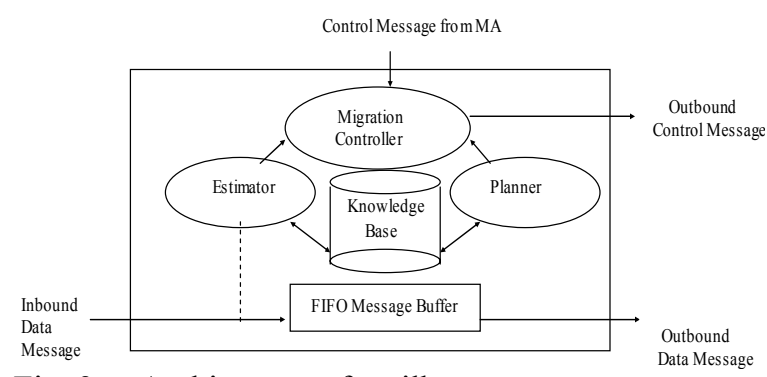

Fig. 3: Architecture of mailbox

knowledge regarding communication environment, the number of message sources, the sender's past behavior. Mailbox is composed of several functional modules as shown in Fig. 3. Estimator module is responsible for tracking history and estimating future message source activities.

Planner module is responsible for itinerary planning. Migration Controller module is responsible for control communication and travel plan execution. Message buffer is used to store incoming message using FIFO policy. Knowledge Base contains knowledge of communication environment. Mailbox makes its predictions of future sender message rates entirely on the information provides by Estimator and Knowledge Base. Reducing the cost of communication by planning optimal mailbox position is the task of Planner. Decision regarding mailbox migration depends on the output generated by Estimator and the knowledge of communication environment. The deployment of simple but efficient planning methods in terms of low resource consumption makes mailbox a lightweight intelligent system. The proactive mailbox is employed to take full advantage of possible cost reduction if message pull occurs at appropriate time such as when mailbox is empty or it contains smaller number of messages.

Mailbox migration: When $\mathrm{MN}$ enters $\mathrm{nFA}$ then it sends an Accommodate message to it. 


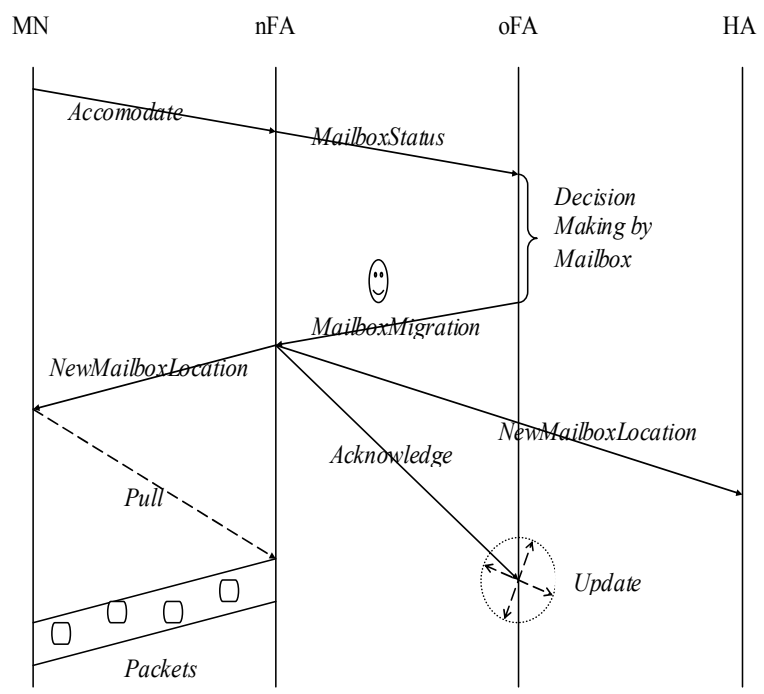

Fig. 4: Activities associated with mailbox migration

The prime information present in this message is address of oFA where mailbox is currently residing. nFA by making the use of this address, sends a MailboxStatus message to oFA as shown in Fig. 4. Upon receiving this message oFA sends a control message to mailbox. Mailbox then makes a decision regarding its migration with the help of its supporting modules.

If mailbox does not migrate then oFA simply record CoA of MN. Otherwise following actions are carried out:

* Mailbox migrates itself to nFA.

* nFA sends an Acknowledge message to oFA.

* In lieu of Acknowledge message oFA sends an Update message to those source hosts which were sending message while mailbox was residing at oFA.

* nFA also sends a NewMailboxLocation message to $\mathrm{HA}$ of MN as well as to MN.

* MN uses a Pull technique to fetch message from mailbox.

Decision logic for mailbox migration: Upon receiving a control message from current MA containing information regarding $\mathrm{nFA}$, the Migration Controller activates a Boolean procedure MBMigration for making a decision regarding its mobility. The pseudo code for this procedure is as follows:

Boolean MBMigration(nFA)

Begin

// $d$ is the distance between $n F A$ and $o F A$

Compute $d=\operatorname{dist}(n F A, o F A)$

Let $n=$ number of messages which will be received by $M N$ at $n F a$ as estimated by Estimator

$/ / d_{T}$ is the threshold value for distance parameter, where $d_{T}=D / 2$ and $D$ is the maximum distance between any two MA in entire network.

// $n_{T}$ is the threshold value for number of messages acting as another

parameter, where $n_{T}=$ sizeof (mailbox)

If $\left(d>d_{T}\right)$ or $\left(n>n_{T}\right)$ then

return true;

else

return false;

End

procedure dist(MAi,MAj) generates the distance between MAi and MAj which is set to the geometric distance in $x-y$ plane.

$$
\mathrm{d}=\sqrt{\left(\left(\mathrm{x}_{\mathrm{i}}-\mathrm{x}_{\mathrm{j}}\right)^{2}+\left(\mathrm{y}_{\mathrm{i}}-\mathrm{y}_{\mathrm{j}}\right)^{2}\right)}
$$

Pull technique: A pull technique, MPUL (Message Pull) is adopted in which a mailbox buffers the messages to its owner $\mathrm{MN}$ and does not need to keep the location information of MN. The MN queries its mailbox periodically for messages. Upon receiving the query message, mailbox forwards one message to $\mathrm{MN}$. If there is no message in mailbox, a null message is sent to $\mathrm{MN}$ as a reply. Query operation can be implemented in synchronous or asynchronous mode. In synchronous mode MN suspends its execution after issuing a query until it receives a reply from its mailbox and as a result of it there would not be any message forwarding to $\mathrm{MN}$ during its migration. In asynchronous mode MN can continue its execution after sending query to its mailbox. MN always knows the location of its mailbox through NewMailboxLocation message as communicated to it by $\mathrm{nFA}$, so location registration is unnecessary in pull technique. Since MN would not leave for next network without receiving the response of its query, there is no message loss or chasing problem in this technique. Pull technique exhibits the following properties:

* Since the MN takes the initiative to request message from its mailbox, the $\mathrm{MN}$ ensures that their would not be any message forwarded to it during its migration thereby resulting in reliable message delivery.

* By querying periodically, MN can easily detect the failure of its mailbox.

* If synchronized query operation is used the MN can leave for next network as soon as it finishes its execution at current network, but execution time is increased. For asynchronous query $\mathrm{MN}$ also has to wait for arrival of all responses of its query before migration. However by deciding the time and number of queries, $\mathrm{MN}$ can flexibly reduce the constraints on its migration.

* Communication overhead depends on the migration and communication pattern of $\mathrm{MN}$. 
* It provides more flexibility as MN has the autonomy to decide the time and frequency of the queries for messages. MN can adjust its query frequency dynamically, if it is in urgent need of information it may query mailbox at a higher frequency. Otherwise a lower frequency is adopted. Distance can be other factor of concern. If current location of $\mathrm{MN}$ is very far from its mailbox, it can query mailbox at lower frequency. When it is near to MA which is holding its mailbox, it can query more frequently.

Performance analysis: Now, we evaluate the total cost incurred during various activities performed at the time of mailbox migration. An assumption is made that all content message are of same size and control message $1 / \sigma$ of content message size. Following parameters are used in the analytical model:

$\sigma-$ ratio between single content message and control message delivery cost $(\mathrm{z}<1)$

$\mathrm{s}_{\mathrm{h}}$-number of source hosts in the system.

$\phi_{\mathrm{MN}}-$ mobile node migration ratio.

$\phi_{\mathrm{M}}-$ mailbox migration ratio.

$\theta_{\mathrm{MN}}-$ mobile node message to migration ratio.

$\theta_{\mathrm{M}}$ - mailbox message to migration ratio.

$\lambda$ - mean message arrival rate i.e. expected number of messages that arrive per unit time.

$\mathrm{t}_{\mathrm{a}}$ - average time period between two adjacent message pulls from mailbox.

$\mathrm{c}_{\mathrm{msg}}$ - average message delivery cost between sender and receiver.

Main components of total communication $\operatorname{cost}\left(\mathrm{c}_{\text {total }}\right)$ are:

$\mathrm{c}_{\text {total }}=\mathrm{c}_{\text {mig }}+\mathrm{c}_{\text {update }}+\mathrm{c}_{\text {pull }}+\mathrm{c}_{\text {MLocation }}+\mathrm{c}_{\text {HAupdate }}$

Cost of mailbox migration $\left(\mathrm{c}_{\text {mig }}\right)$

$=\mathrm{c}_{\text {msg }} \cdot\left(\frac{1}{2 \cdot \mathrm{t}_{\mathrm{a}}} \cdot\left(\mathrm{t}_{\mathrm{a}}-\frac{\mathrm{k}}{\phi_{\mathrm{M}}}\right) \cdot(\mathrm{k}+1)+\frac{\mathrm{k} \cdot(1+\mathrm{k})}{2 \cdot \phi_{\mathrm{M}} \cdot \mathrm{t}_{\mathrm{a}}}\right)$

where $\mathrm{k}=\left\lfloor\mathrm{t}_{\mathrm{a}} \cdot \phi_{\mathrm{M}}\right\rfloor$

Cost of updating source host information regarding mailbox location change ( $\mathrm{c}_{\text {update }}$ ):

$\mathrm{c}_{\text {update }}=\frac{3 \cdot \sigma \cdot \mathrm{c}_{\mathrm{msg}} \cdot \mathrm{s}_{\mathrm{h}}}{\theta_{\mathrm{M}} / \theta_{\mathrm{MN}}}$

Cost incurred while finding new mailbox location by $\operatorname{MN}\left(\mathrm{c}_{\text {MLocation }}\right)$ :
$\mathrm{c}_{\mathrm{MLocation}}=\frac{4 \cdot \sigma \cdot \mathrm{c}_{\mathrm{msg}}}{\lambda \cdot \mathrm{t}_{\mathrm{a}}}$

Cost of MN while pulling all of currently available message in the mailbox $\left(\mathrm{c}_{\text {pull }}\right)$ :

$\mathrm{c}_{\text {pull }}=\mathrm{c}_{\text {mgg }}+\frac{\sigma \cdot \mathrm{c}_{\text {mgg. }}\left(1+\mathrm{e}^{-\lambda \mathrm{t}_{\mathrm{a}}}\right)}{\lambda \cdot \mathrm{t}_{\mathrm{a}}}$

Cost of updating current mailbox or MN position at the corresponding HA $\left(\mathrm{c}_{\text {HAupdate }}\right)$ :

$\mathrm{c}_{\text {HAupdate }}=\frac{\sigma \cdot \mathrm{c}_{\mathrm{msg}}}{\theta_{\mathrm{M} / \theta_{\mathrm{MN}}}}$

Some research results on performance analysis of Mobile IP can be found in ${ }^{[10-13]}$.

Related work: A seamless streaming framework is introduced in $^{[14]}$ by estimating the accurate buffer level for pre-buffering to compensate the handoff latency under Mobile IPv4 environment. An enhanced buffer management mechanism for fast handover is implemented $\mathrm{in}^{[15]}$ but still based on traditional tunneling establishment. Performing smooth handoff in a model resembling ours was also considered $\mathrm{in}^{[16,17]}$. The decision regarding mailbox migration was forced on mobility agents in these models, unlike ours where the presence of knowledge base and other supporting modules in a mailbox enriches it with the decision making capability regarding its migration thereby making it an intelligent component.

\section{CONCLUSION}

The proposed intelligent mailbox can itself makes a decision regarding its de coupling from owner mobile node considering both the distance as well as network traffic between source and target mobility agents thereby resulting in the reduction in packet loss and workload on home agent. Asynchrony is improved in two aspects. Firstly with address caching mechanism the reliance on home agent for node tracking and message forwarding is reduced. Secondly, the constraint on node mobility is released because synchronization occurs between source nodes and mailbox, MN can migrate to new network whenever it wants without waiting for messages in transits. The use of pull technique for transfer of message from mailbox to mobile node reduces network traffic and delay of message processing. 


\section{REFERENCES}

1. Perkins, C. and D. Johnson, 2004. Mobility Support in IPv6. RFC 3775, IETF.

2. Perkins, C.E., 2002. IP Mobility Support for IPv4. RFC3344.

3. Dixit, S. and R. Prasad, 2002. Wireless IP and Building the Mobile Internet. Atrech House, Incorporated, pp: 393-413.

4. Hsieh, R., Z. Zhou and A. Seneviratne, 2003. SMIP: A seamless handoff architecture for mobile IP. IEEE, INFOCOM 2003.

5. Yana, B., M. Song and J. Song, 2005. Seamless mobility using mobile IPv6. 2nd Intl. Conf. on Mobile Technology, Applications and Systems, pp: 1-8.

6. Vivaldi, L., B.M. Ali, H. Habaebi, V. Prakash and A. Sali, 2003. Routing scheme for macro mobility handover in hirarchical mobile IPv6 network. Proc. 4th Natl. Conf. on Telecommunication Technology (NCTT 2003), pp: 88-92, 14-15 Jan.

7. Cho, H., T.Y. Kwon and Y.G. Choi, 2006. Route optimization using tree information for nested mobile networks. IEEE J. on Selected Areas in Communications, 24: 9.

8. Shaojian Fu, Liran Ma, Atiquzzaman M., Yong-Jin Lee," Architecture and performance of SIGMA: a seamless mobility architecture for data networks",IEEE International Conference on Communications,ICC 2005,pp:3249-3253,Vol.5, May 2005.

9. Berezdivin, R., R. Breining and R. Top, 2002. Next-generation wireless communications concepts and technologies. IEEE Commun. Mag., 40: 04-105.
10. Hsieh, R. and A. Seneviratne, 2003. A comparison of mechanisms for improving mobile IP handoff latency for end-to-end TCP. Proc. of 9th Annu. Intl. Conf. on Mobile Computing and Networking, pp: $29-41$.

11. Perkins, C. and K.Y. Wang, 1999. Optimized smooth handoffs in Mobile IP. Proc. IEEE Symp. on Computers and Communications.

12. Patricia, K.B. and R. Pendse, 2006. Quantitative analysis of enhanced mobile IP. IEEE Commun. Mag., 44: 66-72.

13. Wu, J.C.S., C.W. Cheng, G.K. Ma and N.F. Huang, 2001. Intelligent handoff for mobile wireless internet. Mobile Networks and Applications, pp: 67-79.

14. Dongwook, L0, C.-H. Lee and J.W. Kim, 2005. Seamless media streaming over mobile IP-enabled wireless LAN. 2nd IEEE Consumer Communications and Networking Conf., pp: 116121.

15. Krishnamurthi, G., R.C. Chalmers and C.E. Perkins, 2001. Buffer management for smooth handover in IPv6. Internet Draft, IETF.

16. Cao, J., L. Zhang, H. Chan and S.K. Das, 2004. Design and performance evaluation of an improved mobile IP protocol. IEEE, INFOCOM, pp: 329.

17. Feng, X., J. Cao, J. Lu and H. Chan, 2001. An efficient mailbox based algorithm for message delivery in mobile agent systems. Proc. of 5th IEEE Intl. Conf. on Mobile Agents, LNCS 2240, pp: $135-151$. 